\title{
Intoxicación por paraquat: presentación de dos casos y revisión de la literatura
}

\author{
A. RIVERO GONZÁLEZ, J.F. NAVARRO GONZÁLEZ, M.L. MACÍA HERAS, \\ C. MORA FERNÁNDEZ, M.L. RODRÍGUEZ SERRANO, J. GARCÍA PÉREZ
}

Servicio de Nefrología. Hospital Nuestra Señora de Candelaria. Santa Cruz de Tenerife

\author{
PARAQUAT POISONING: REPORT OF TWO CASES AND LITERATU - \\ RE REVIEW
}

\begin{abstract}
RESUMEN
La intoxicación por pesticidas, especialmente con fines suicidas, continua siendo un importante problema terapéutico. El herbicida paraquat (1,1' dimetil-4,4' bipiridilo dicloro) es el $2^{\circ}$ agente causal de intoxicación por pesticidas en nuestro país, estando asociada a una alta tasa de mortalidad. Presentamos dos casos de intoxicación por paraquat con fines autolíticos que desarrollaron fracaso multiorgánico y tuvieron una evolución fatal. Presentamos también una breve revisión de la literatura centrada principalmente en las diferentes opciones terapéuticas.
\end{abstract}

PALABRAS CLAVE: Paraquat. Intoxicación. Terapéutica.

\begin{abstract}
Cases of poisoning with pesticides, especially suicidal ones, conti nue to be an important therapeutic problem. The heribicide paraquat (1.1' dimethyl-4.4' bipyridylium dichloride) is the second cause of pesti cide poisoning in our country, which is associated with a high mortality rate. We report two cases of suicidal ingestion of paraquat who develo ped multiorgan failure with a lethal outcome. We also present a brief review of the literature, mainly focused on the different therapeutic options.
\end{abstract}

KEY WORDS: Paraquat. Poisoning. Therapeutics.

Rivero González A, Navarro González JF, Macía Heras ML, Mora Fernández C, Rodríguez Serrano ML, García Pérez J. Intoxicación por paraquat: presentación de dos casos y revision de la literatura. An Med Interna (Madrid) 2001; 18: 208-210.

\section{INTRODUCCIÓN}

El paraquat es un potente herbicida de contacto del grupo bipiridílico de amplia utilización en la agricultura. Aunque se han descrito casos de intoxicación por vía cutánea, conjuntival e incluso parenteral (1), las intoxicaciones más frecuentes por este compuesto se producen debido a su ingesta de forma voluntaria con fines autolíticos. La afectación que produce en nuestro organismo se ha comprobado prácticamente a todos los niveles (2), pero destaca de forma especial el compromiso pulmonar, responsable de gran parte de los casos de fallecimiento.

Desde un punto de vista epidemiológico es en los países del Lejano Oriente donde se produce el mayor número de intoxicaciones por estos productos. Es importante destacar que en nuestro país los herbicidas son, tras los insecticidas organofosforados, la segunda causa de intoxicación por pesticidas (3). La importancia de la intoxicación por estos productos deriva de su alta frecuencia, del alto porcentaje de mortali- dad que conlleva y de la escasa eficacia de los tratamientos existentes. Presentamos dos casos de intoxicación por paraquat y hacemos una revisión de la literatura con especial atención a los aspectos terapéuticos.

\section{CASOSAPORTADOS}

Caso 1: Varón de 31 años, sin antecedentes de interés excepto fumador moderado, que ingresa en el servicio de urgencias tras haber ingerido de forma voluntaria, aproximadamente 14 horas antes, unos $100 \mathrm{cc}$. de Gramaxone ${ }^{\circledR}$ extra al $20 \%$. El paciente refiere dolor abdominal, molestias en cavidad bucal e intensos vómitos. A la exploración física inicial el paciente se encontraba consciente y orientado témporo-espacialmente, normotenso $(140 / 80 \mathrm{mmHg})$, con una frecuencia cardiaca de 94 latidos por minuto y una temperatura axilar de $37,3^{\circ} \mathrm{C}$. La exploración de la cavidad oral reveló la existencia de lesiones eritematosas en orofaringe. El abdomen era doloroso a la palpación, sin defensa ni signos de irritación peritoneal. En los estudios de laboratorio destacaban: hemoglobina $15 \mathrm{~g} / \mathrm{dl}$, hematocrito $46 \%$,

Trabajo aceptado: 17 de Agosto de 1999

Correspondencia: A. Rivero González. Servicio de Nefrología. Hospital Nuestra Señora de Candelaria. Ctra. del Rosario s/n. 38010 Santa Cruz de Tenerife. 
leucocitos $14.500 / \mathrm{mm}^{3}$ (84 \% neutrófilos), plaquetas $240000 / \mathrm{mm}^{3}$, glucosa $200 \mathrm{mg} / \mathrm{dl}$, GOT $78 \mathrm{U} / \mathrm{L}$, GPT $91 \mathrm{U} / \mathrm{L}$, creatinina $2,1 \mathrm{mg} / \mathrm{dl}$. El ionograma, los tiempos de protrombina y el tiempo de cefalina se encontraban dentro de límites normales, así como los gases arteriales y el equilibrio ácido-base. En la radiografía de tórax destacaba un leve refuerzo de la trama bronquial. La determinación de ditionita sódica fue positiva. Inicialmente además de sueroterapia se administró manitol al 20\%, carbón activado y tierra de Füller por SNG, así como vitamina $\mathrm{C}$ y protectores gástricos. El paciente fue trasladado a la Unidad de Cuidados Intensivos donde se inició hemoperfusión con cartuchos de carbón activado. Tras la primera sesión sufrió un episodio de hipotensión arterial severa que requirió tratamiento con aminas vasoactivas. Posteriormente desarrolló un cuadro de hematemesis y melenas. Durante una nueva sesión de hemoperfusión se produjo parada respiratoria que obligó a intubación orotraqueal y ventilación mecánica. En las horas siguientes presentó gran inestabilidad hemodinámica y parada cardiaca por disociación electromecánica que fue refractaria a todas las medidas terapéuticas, produciéndose el exitus tras 12 horas de ingreso.

Caso 2: Paciente varón de 45 años de edad con antecedentes de etilismo crónico que ingresa en el Servicio de Urgencias tras la ingestión de Gramaxone ${ }^{\circledR}$ extra al 20\%. El tiempo transcurrido desde la ingesta del herbicida así como la cantidad ingerida no fueron precisados. A la exploración física el paciente aparace obnubilado, taquipneico, taquicárdico, moderadamente deshidratado y con lesiones eritematosas en la mucosa oral. Los estudios analíticos revelaron un hemograma normal, glucosa $135 \mathrm{mg} / \mathrm{dl}$ y creatinina $1,5 \mathrm{mg} / \mathrm{dl}$. El resto de determinaciones bioquímicas incluyendo transaminasas e iones, así como el equilibrio ácido-base eran normales. La determinación de ditionita sódica fue positiva. La radiografía de tórax no mostró datos significativos. Se administró sueroterapia, manitol al $20 \%$, carbón activado, sulfato de Mg y tierra de Füller, pasando a continuación a la Unidad de Cuidados Intensivos donde se procedió a intubación y ventilación mecánica. Posteriormente presentó deterioro hemodinámico con buena respuesta inicial a expansores de plasma y aminas vasoactivas. En las horas siguientes presenta fiebre de $40{ }^{\circ} \mathrm{C}$ y fallo multiorgánico con compromiso predominantemente pulmonar y renal sin respuesta al tratamiento que incluyó administración de antioxidantes y corticosteroides. Después de 8 horas de ingreso el paciente fallece por desarrollo de trastornos cardiacos.

\section{DISCUSIÓN}

En los casos presentados destaca una rápida y desfavorable evolución hacia el exitus en menos de 72 horas a pesar de las medidas terapéuticas instauradas, lo cual ilustra la gravedad de la intoxicación por este tipo de herbicidas.

Los clásicos trabajos de Proudfoot et al $(4,5)$ permitieron establecer el pronóstico de la intoxicación por paraquat en relación con dos factores principales: el tiempo transcurrido desde la ingesta y la concentración plasmática del tóxico. Sin embargo, en muchas ocasiones su valor práctico se encuentra limitado por circunstancias como no conocer la cantidad ingerida del herbicida y el tiempo desde su ingestión, así como no existir determinaciones rutinarias de esta sustancia en muchos centros. Se han descrito tres formas clínicas en función de la severidad del cuadro y de la cantidad de tóxico ingerida: a) intoxicación fulminante, cuando la cantidad ingerida de tóxico es mayor de $50 \mathrm{mg} / \mathrm{kg}$ de peso, produciéndose la muerte en menos de 72 horas por fallo multisistémico; b) intoxicación moderada-severa, cuando se ingieren de 20 a $40 \mathrm{mg} / \mathrm{kg}$ de peso. Esta forma cursa con una primera fase donde predomina la clínica gastrointestinal, seguida del desarrollo de fallo hepato-renal entre el $2^{\circ}$ y el $5^{\circ}$ día. Después de unos 7 días aparece un cuadro de distress respiratorio y finalmente acontece la muerte entre la $2^{\mathrm{a}}$ y $4^{\mathrm{a}}$ semana; c) intoxicación leve, cuando la cantidad ingerida es menor de $20 \mathrm{mg} / \mathrm{kg}$ de peso. Esta forma sólo presenta clínica gastrointestinal y alteración de las pruebas funcionales respiratorias $(6,7)$.

El tratamiento de la intoxicación por paraquat presenta la importante limitación de no contar con un antídoto específico. Por ello, es de especial importancia la rapidez en la instauración de medidas destinadas a evitar la absorción del tóxico, a facilitar su extracción del plasma y su eliminación por la orina y a neutralizar sus efectos a nivel de órganos diana. La revisión de la literatura con relación al tratamiento de estas intoxicaciones revela que son numerosas las medidas terapéuticas empleadas, pero al mismo tiempo se observa una gran controversia en cuanto a los resultados obtenidos.

Para evitar la absorción digestiva de este herbicida se han utilizado catárticos asociados a adsorbentes, así como resinas de intercambio iónico con una alta capacidad de fijación para el paraquat (8). Recientes estudios en modelos animales han demostrado que compuestos como los alquilsulfatos y alquilsulfonatos pueden tener una efectiva capacidad detoxicante frente al paraquat, reduciendo su absorción a nivel intestinal además de inhibir la formación de complejos lipídicos a nivel pulmonar (9). Actualmente, el desarrollo de nuevas opciones terapéuticas se centra en el estudio de los mecanismos intrínsecos de la absorción digestiva de este herbicida.

En lo referente a la extracción del tóxico del plasma una vez que se ha producido su absorción, es de destacar el papel de las técnicas de depuración extrarrenal. Las pautas intensivas de hemoperfusión han demostrado su utilidad en algunos casos (10), incluso en situaciones de intoxicación masiva, donde el empleo de estas técnicas junto a productos como la desferroxamina y otros antioxidantes han tenido resultados exitosos. A pesar de ello, diversos autores discuten su utilidad sobre la base de las características farmacocinéticas del paraquat: gran volumen de distribución, rápida desaparición de sus niveles sanguíneos y lenta transferencia intercompartimental $(11,12)$.

Pero sin duda, el mayor interés en el tratamiento de estas intoxicaciones se ha centrado en las medidas encaminadas a neutralizar sus efectos a nivel de los diferentes órganos diana. La acción tóxica del paraquat a nivel hístico se debe a la reducción-reoxidación cíclica del catión bipiridílico con formación de radicales libres, principalmente en los neumocitos tipo II. Esta reacción produce una deplección de diversos enzimas como el glutation, la superóxido dismutasa y el cofactor NADPH, importantes en su papel como antioxidantes, lo cual resulta en la afectación de las membranas celulares. En este escenario se han utilizado ácidos grasos monoinsaturados como el ácido oleico (13), beta-bloqueantes, por su supuesta capacidad de competir con los receptores pulmonares del paraquat (14), antioxidantes como la $\mathrm{N}$ - acetil-cisteína o las vitaminas E y C, y enzimas como la superóxido dismutasa vehiculada en liposomas (15). Otras opciones terapéuticas sugeridas recientemente incluyen la melatonina, capaz de reducir la capacidad oxidativa del herbicida disminuyendo así la cantidad de glutation oxidado (16), el litoespermato magnésico, al reducir la toxicidad renal y mejorar la eliminación del tóxico (17), e incluso anticuerpos específicos dirigidos contra el paraquat, los cuales son capaces de inhibir la 
captación del herbicida por el neumocito tipo II (18). Por último, las medidas dirigidas a reducir la reacción inflamatoria a nivel tisular y a tratar el distress respiratorio han sido consideradas en el tratamiento de esta intoxicación. Dentro de las primeras se han utilizado corticoesteroides, solos o asociados a inmunosupresores en un intento de reducir la alveolitis y la posterior fibrosis (19). Asimismo, para inhibir la profileración fibroblástica desencadenada por el tóxico se ha usado la radioterapia (20), aunque los resultados son controvertidos. Entre las medidas destinadas a combatir el distress respiratorio destaca el empleo del óxido nítrico, tras cuya administración se ha objetivado una mejoría de la presión arterial pulmonar y un incremento de la presión parcial de oxígeno (21).

Finalmente, cuando estas medidas fracasan y se establece la lesión pulmonar, el trasplante ha comenzado a demostrar su validez como alternativa terapéutica. Hasta 1996 los intentos de trasplante pulmonar en pacientes en situación de insuficiencia respiratoria terminal debido a esta intoxicación habían fracasado. Sin embargo, recientemente Walder y cols. (22) han presentado el primer trasplante pulmonar con éxito en un paciente después de la intoxicación por paraquat.

\section{Bibliografía}

1. Alvarez B, Rosado L, Berenggguer J. Intoxicación aguda via intravenosa con paraquat. Medicina Intensiva 1998; 12: 343-345.

2. Palomar M."Herbicidas". En Marruecos: L Nogue S, Nolla J.editores. Toxicología clínica Barcelona: Springer Velag Ibérica S.A. 1993; 255 265.

3. Rodriguez Pozo A, Martín Rubi JC, Martín Delgado MC, Alonso Rubio M. Grupo de trabajo de Toxicología de la S.E.M.I.U.C. Intoxicaciones agudas por pesticidas. Estudio multicéntrico naccional de 10 años (resultados preliminares). Medicina Intensiva 1996; 20: 1.

4. Proudfoot AT, Stewart MS, Levitt T, Widdop B. Paraquat poisoning: significance of plasma paraquat concentrations. Lancet 1979; 2: 330-332.

5. Hart TB, Nevitt A. A new statistical approach to the prognostic significance of plsma paraquat concentration. Lancet 1984; 2: 1222-1223.

6. Mencías E, Cano A, Cabrera R. Intoxicación por Paraquat. JANO 1993; 1029: 839-350.

7. Nuñez R, Sanchez MC, Intoxicación por productos agrícolas. En: Ginestal RJ, et al.Cuidados Intensivos (Madrid). Ed. ELA 1991; 15931599.

8. Yamashita M, Naito H, Tadagi S. The effectiviness of a cation resin (kayexolate) as an adsorbeent of paraquat: experimental and cliniccal studies. Human Toxicol 1987; 6: 89-90.

9. Tsuchiya T, Yoshida T, Imaeda A, Kiho T, Ukai S. Detoxificacion of paraquat poisoning: effects of alkylsulfates and alkylsulfonates on paraquat poisoning in mice an rats.Biol Pharm Bull 1995; 18: 523-528.

10. Suzuki K, Takasu N, Okabe T, Ishimatsu S, Ueda A, TnakaS, Fukuda A et al. Effect aggressive haemoperfusion on the clinical course patiens with paraquat poisoning. Hum Exp Toxicol 1993; 12: 323-327.

11. Bismuth C, Scherrmann JM, Garnier R, Baud FJ, PONTAL pg. Elimination of paraquat. Human Toxicol 1987; 6: 63-67.
12. Proudfoot AT, Prescott LF, Jarvie DR. Haemodialysis for paraquat poisoning. Human Toxicol 1987; 6: 69-74.

13. Fritz K, Nelson TL, Ruiz Velasco V, Mecurio SD. Acute intramuscular injection of oil acid component protects mice against paraquat lethality. J Nuttr 1994; 124: 4255-429.

14. Fairsther RD, Rosen SM, Smith WR, Glauser FL, Mc Rae DM, Wilson AF. Paraquat poisoning: new aspect of therapy. QJ Med 1976; 180: 551-565.

15. Bateman DN. Pharmacological treatment of paraquat poisooning. Human Toxicol 1987; 6: 52-62.

16. Melchiorri D, Reiter RJ, Sewerynek E, Chen L, Nistico G. Paraquat toxicity and oxidative damage. Reduction by melatonin. Biochemical Pharmacology 1996; 51: 1095-1099.

17. Takako Yokozawa, Hikokichi Oura, Itsuo Nishioka. Confirmation that Magnesium Lithospermate B ameliorates paraquat induced injury in cultured renal epithelial cells. Nephron 1998; 79: 373-374.

18. ChenN, Borules MR, Pond SM. Prevention of paraquat toxicity in suspensions of alveolar type II by paraquat specific antibodies. Hum Exp Toxicol 1994; 13: 5511-557.

19. Ja Liang Lin, Mein Chung Wei, Yu Chih Liu. Pulse therapy with cyclophosphamide and methylprednisolone in patiens with moderate to severe paraquat poisoning: a prelimary repot. Thorax 1996; 51: 661-663.

20. William HV, Webb DB. paraquat lung: is ther a role for radiotherapy? Human Toxicol 1987; 6: 75-81.

21. KoppelC, von Wissman C, Barkckow d et al. Inhaled nitric oxide in advanced paraquat intoxication. J Toxicol Clin Toxicol 1994; 32: 205-214.

22. Bernhard Walder, Marie Anne Brüdler, Anastase Spiliopoulos, Jacques A. Romand. Successful single lung transplantation after paraquat intoxication. Transplantation 1997; 64: 789-791. 